\title{
Neutrino Oscillations Founded on Tachyon Theory of Neutrino
}

\author{
Zoran B. Todorovic \\ Faculty of Sciences, Department of Physics, University of Pristina, Kos. Mitrovica, Serbia
}

Email address

dgtzoranpw@yahoo.com

To cite this article:

Zoran B. Todorovic. Neutrino Oscillations Founded on Tachyon Theory of Neutrino. International Journal of Astrophysics and Space

Science. Special Issue: Quantum Vacuum, Fundamental Arena of the Universe: Models, Applications and Perspectives.

Vol. 2, No. 6-1, 2014, pp. 18-32. doi: 10.11648/j.ijass.s.2014020601.13

\begin{abstract}
De Broglie's neutrino relations have been derived in this paper in the spirit of tachyon neutrino theory. The analysis of the physical characteristics of the neutrino as the tachyon particle has been done and a formula derived for the oscillation length. By analyzing phase angle of the plane wave, we came to the conclusion that the oscillations of neutrinos could be performed by speeds greater than the speed of light. Starting off from the application of Heisenberg's uncertainty relation in the micro-world, the postulate of neutrino confinement was introduced in the macroscopic area defined by the neutrino oscillation length. It is shown that the neutrino mass which belongs to the tachyon four-dimension space-time and the neutrino mass of the four-dimension space-time of the theory of relativity are not mutually equal by value, but the corresponding energy and momentum are unchanging.
\end{abstract}

Keywords: Ordinary Neutrino, Tachyon, Neutrino Oscillations, Oscillation Length

\section{Introduction}

In this work we shall try on to find out the answer on only one question which is defined as in $[1,2]$. The question is posted in the following form:" Why do the often used sameenergy and same-momentum assumption for neutrino mass eigenstates composing a given flavor state which are known to be both wrong, lead to the correct result for the oscillation probability?" In order to give the answer on posted question we shall found our investigation on the condition which is related with the case of same- energy assumption, The content of this paper is occupied with the several sections which are tilted as follows: 2 . Definition of tachyon velocity. 3. De Broglie's relations for tachyons.4. Two flavor oscillations 5. Klein-Gordon equation for neutrinos. 6. Free particle solutions. 7. Why neutrinos as tachyons mutually oscillate? 8. Approximate relations. 9. Proceeding for two flavor oscillations in the case of equal-energy assumption. 10 . Postulate of neutrino flavor confinement. 11. The ratio between neutrino masses. 12. The ratio between neutrino velocities. 13. The ratio between neutrino momentums. 14 . The plane wave or the wave packet? 15. Transformation between two different four-dimensional space-times. 16. Comment on the oscillation phase. 17. Conclusion. As an example of our investigation is neutrino velocity which can be represented as a product of wavelength and frequency. Especially an attention has been dedicated to the phase factor of neutrino plane wave. On the basis of derived formula which belongs to oscillation length has been shown a connection between it and physical characteristic of neutrino. And then are given the conditions under which are possible to occur neutrino oscillations .On the basis of Heisenberg's uncertainty relation has been defined the postulate about the flavor neutrino confinement which connects on the unique way macroscopic characteristic-oscillation length with neutrino features as a quantum-mechanical particle.

\section{Definition of Tachyon Velocity}

In the empty space neutrino moves at $U=c(1+\delta)>c ; \delta>0$ and it connects its momentum $p$ with energy $E$ by relation as in $[3,4]$

$$
E=p U
$$

where $p$ is the magnitude of the momentum vector $\vec{p}$.The energy of neutrino in explicit form is described by tachyon energetic right-angle triangle as in $[3,4]$ 


$$
E^{2}=\left(p \frac{U^{2}}{c}\right)^{2}+\left(i m U^{2}\right)^{2} ; i=\sqrt{-1}
$$

or in the form:

$$
\begin{aligned}
& E=m \Gamma U^{2}=\frac{U^{2}}{c} \sqrt{p^{2}-m^{2} c^{2}} \\
& =p U
\end{aligned}
$$

Here $\Gamma$ is tachyon factor

$$
\Gamma=\frac{1}{\sqrt{U^{2} / c^{2}-1}}
$$

And there is one more neutrino's energy relation

$$
\begin{aligned}
& E=m \Gamma U^{2}=c \sqrt{p^{2}+m^{2} U^{2}} \\
& =p U
\end{aligned}
$$

The momentum of neutrino is in the following relation with its energy:

$$
\begin{aligned}
& p=m \Gamma U=\frac{U}{c} \sqrt{\frac{E^{2}}{U^{2}}-m^{2} c^{2}} \\
& =\frac{1}{c} \sqrt{E^{2}-m^{2} c^{2} U^{2}}
\end{aligned}
$$

In order to obtain neutrino velocity we shall use the following expressions:

$$
\begin{aligned}
& m^{2} c^{2}=\frac{p^{2}}{(1+\delta)^{2} \Gamma^{2}} \\
& m^{2} U^{2}=\frac{p^{2}}{\Gamma^{2}} \\
& U=c \sqrt{1+\frac{1}{\Gamma^{2}}}
\end{aligned}
$$

Introducing the relations $(6,7,7 \mathrm{a}, 8)$ within the energy relations (2) and (4) they become:

$$
\begin{gathered}
E=\frac{U^{2}}{c} \sqrt{p^{2}-m^{2} c^{2}} \\
=\frac{U^{2}}{c} p \sqrt{1-\frac{1}{(1+\delta)^{2} \Gamma^{2}}} \\
E=c \sqrt{p^{2}+m^{2} U^{2}}=p c \sqrt{1+\frac{1}{\Gamma^{2}}}
\end{gathered}
$$

By differentiation relations (9) and (10) per its momentum $p$ :

$$
\begin{aligned}
& \frac{\partial E}{\partial p}=\frac{\partial}{\partial p}\left[\frac{U^{2}}{c} \sqrt{p^{2}-m^{2} c^{2}}\right] \\
& =\frac{U^{2}}{c} \sqrt{1-\frac{1}{(1+\delta)^{2} \Gamma^{2}}}=\frac{U^{2}}{c(1+\delta)}=U
\end{aligned}
$$

where

$$
U=c \frac{1}{\sqrt{1-\frac{m^{2} c^{2}}{p^{2}}}} \approx c\left(1+\frac{m^{2} c^{2}}{2 p^{2}}\right)>c
$$

Also,

$$
\frac{\partial E}{\partial p}=\frac{\partial}{\partial p}\left[c \sqrt{p^{2}+m^{2} U^{2}}\right]=c \sqrt{1+\frac{1}{\Gamma^{2}}}=U
$$

where

$$
\begin{aligned}
& \frac{p^{2} U^{2}}{c^{2}}-m^{2} U^{2}=p^{2} \\
& U=c \frac{1}{\sqrt{1-\frac{m^{2} c^{2}}{p^{2}}}} \approx c\left(1+\frac{m^{2} c^{2}}{2 p^{2}}\right)>c
\end{aligned}
$$

ones get the same magnitude of neutrino velocity. Despite of the direct differentiation in theory of relativity

$$
\frac{\partial E}{\partial p}=\frac{\partial}{\partial p}\left[c \sqrt{p^{2}+m^{2} c^{2}}\right]=\frac{c^{2} p}{E}
$$

which is allowed because of the functional dependence between energy and momentum, that is not allowed in tachyon theory because of the complex dependence between energy and momentum, as it is shown by relations (3) and (5).One more reason of that is the fact itself that in theory of relativity there are rest energy and rest mass, but in tachyon theory the particles do not possess rest mass nor rest energy.

\section{De-Broglie's Relations for Neutrinos as Tachyons}

Similarly as for photon, the energy and the neutrino momentum depend from frequency $f$ and, from the wavelength $\lambda$ :

$$
\begin{gathered}
E=\hbar \omega=h f=p U \\
\vec{p}=\hbar \vec{k}=\hbar \frac{2 \pi}{\lambda} \vec{e}_{k}=\frac{h}{\lambda} \vec{e}_{k} ;\left(\vec{e}_{k}=\frac{\vec{k}}{|\vec{k}|}\right)
\end{gathered}
$$

On the basis of relations (3) i (5) one finds

$$
E=h f=h \frac{U}{\lambda}
$$

and from here immediately follows:

$$
U=\lambda f
$$

That the neutrino speed is equal to the product of wavelength and frequency. And the magnitude of wave vector $\vec{k}$ defines its wave number 


$$
k=|\vec{k}|=\frac{2 \pi}{\lambda}=\frac{\omega}{U}
$$

The neutrino momentum $\vec{p}$ is directed along its line of propagation, till its magnitude amounts

$$
p=\hbar k=\frac{h}{\lambda}=\frac{h f}{U}=\frac{E}{U}
$$

Where $h$ is Planck's constant

If we apply a rule in the equation of motion that the distance is equal to the product of either velocity and time ( dis $\tan c e=$ velocity $\times$ time $)$ on the wavelength $\lambda$ as a distance, then the wavelength is equal to the product of either neutrino speed $U$ and time $T$

$$
\lambda=U T
$$

Taking into account that

$$
f=\frac{1}{T}
$$

one gets neutrino speed

$$
U=\lambda f
$$

Where $f=$ frequency; $T=$ period .

Introducing de Broglie's relations for the momentum and the speed within equation (3) we have:

$$
\begin{aligned}
& E=m \Gamma U^{2}=\frac{U^{2}}{c} \sqrt{p^{2}-m^{2} c^{2}} \\
& =\frac{\lambda^{2} f^{2}}{c} \sqrt{\frac{h^{2}}{\lambda^{2}}-\frac{p^{2}}{\Gamma^{2} U^{2}} c^{2}}=h f
\end{aligned}
$$

and for the momentum

$$
\begin{aligned}
& p=m \Gamma U=\frac{c E}{U^{2}} \sqrt{1+\frac{1}{\Gamma^{2}}} \\
& =E \sqrt{\frac{1}{c^{2}}-\frac{1}{\Gamma^{2} U^{2}}}=\frac{h}{\lambda}
\end{aligned}
$$

In the case of equal energies of two different types of tachyons

$$
E_{1}=p_{1} U_{1}=p_{2} U_{2}=E_{2}=E=h f
$$

if we separately calculate $p_{1}$

$$
\begin{aligned}
& p_{1}=m_{1} \Gamma_{1} U_{1} \\
& \approx m_{1} \frac{1}{\sqrt{2 \delta_{1}}} \lambda_{1} f=\frac{h}{\lambda_{1}}
\end{aligned}
$$

we shall get :

$$
\begin{aligned}
& p_{1} \lambda_{1}=m_{1} \frac{1}{\sqrt{2 \delta_{1}}} \lambda_{1}^{2} f=h ; \\
& \lambda_{1} f=c\left(1+\delta_{1}\right) \approx c ; \delta_{1}<<1
\end{aligned}
$$

And for $p_{2}$ will be:

$$
\begin{aligned}
& p_{2} \lambda_{2}=m_{2} \Gamma_{2} U_{2} \lambda_{2} \approx m_{2} \frac{1}{\sqrt{2 \delta_{2}}} \lambda_{2}^{2} f=h ; \\
& \lambda_{2} f=c\left(1+\delta_{2}\right) \approx c, \delta_{2}<<1
\end{aligned}
$$

where as in $[4,25]$

$$
\begin{gathered}
\delta_{1} \approx \frac{m_{1}^{2} c^{4}}{2 E_{1}^{2}} \\
\delta_{2} \approx \frac{m_{2}^{2} c^{4}}{2 E_{2}^{2}}
\end{gathered}
$$

Equating (26) with (27) we have:

$$
\begin{aligned}
& p_{1} \lambda_{1}=m_{1} \frac{1}{\sqrt{2 \delta_{1}}} \lambda_{1}^{2} f \\
& p_{2} \lambda_{2}=m_{2} \frac{1}{\sqrt{2 \delta_{2}}} \lambda_{2}^{2} f
\end{aligned}
$$

And from (30) one gets:

$$
\frac{m_{1}}{m_{2}}=\frac{\lambda_{2}^{2}}{\lambda_{1}^{2}} \sqrt{\frac{\delta_{1}}{\delta_{2}}}
$$

Suppose that the tachyon energy $E$ is far above from its minimum of energetic function at the its left side. The speed in its left section can then be represented by the approximation:

$$
\begin{aligned}
& u_{\delta}=c(1+\delta) \approx c\left(1+\frac{m^{2} c^{4}}{2 E^{2}}\right)=\lambda_{\delta} f \\
& \delta>0 ; \delta<<1
\end{aligned}
$$

and its wavelength is

$$
\lambda_{\delta} \approx \frac{c}{f}
$$

But, because of the shape of the energetic function (3), which we were show in reference [4], that there is, for the same energy $E$, the speed placed at the right section equal to

$$
u_{n \delta}=c(1+n \delta) \approx \frac{c}{\sqrt{2 \delta}}=\lambda_{n \delta} f ; n \delta>>1
$$

Introducing the value for $\delta(28,29)$

$$
\delta=\frac{m^{2} c^{4}}{2 E^{2}}
$$

one gets

$$
u_{n \delta}=\frac{c}{\sqrt{2 \frac{m^{2} c^{4}}{2 E^{2}}}}=\frac{E}{m c}=\lambda_{n \delta} f
$$


And the wavelength $\lambda_{n}$ is:

$$
\lambda_{n \delta} \approx \frac{h}{m c}
$$

Because the momentum at $n \delta \gg 1$ or, $n \delta \rightarrow \infty$ equal to

$$
\begin{aligned}
& p_{n \delta \rightarrow \infty}=\lim _{n \delta \rightarrow \infty} p=\lim _{U \rightarrow \infty} m \Gamma U \\
& =\lim _{U \rightarrow \infty} m \frac{1}{\sqrt{U^{2} / c^{2}-1}} U=m c
\end{aligned}
$$

So, we have, for the same energy $E$, two possible wavelengths of the tachyon $\lambda_{\delta}$ and $\lambda_{n \delta}$. Namely, the relation between (33) and (37) gives:

$$
\frac{\lambda_{n \delta}}{\lambda_{\delta}} \approx \frac{h / m c}{c / f}=\frac{h f}{m c^{2}}=\frac{E}{m c^{2}}
$$

Energy of the left section of the energy function (3) is

$$
E=m \Gamma_{\delta} U_{\delta}^{2}=m \frac{(1+\delta)^{2}}{\sqrt{(1+\delta)^{2}-1}} c^{2} \approx m \frac{1}{\sqrt{2 \delta}} c^{2} ; \delta<<1
$$

Also, for the right section of the same energy function we can write:

$$
\begin{aligned}
& E=m \Gamma_{n \delta} U_{n \delta}^{2}=m \frac{(1+n \delta)^{2}}{\sqrt{(1+n \delta)^{2}-1}} c^{2} \\
& \approx m c^{2}(1+n \delta) \approx m c^{2} \frac{1}{\sqrt{2 \delta}}
\end{aligned}
$$

Then relation (39) becomes:

$$
\frac{\lambda_{n \delta}}{\lambda_{\delta}} \approx \frac{h f}{m c^{2}}=\frac{E}{m c^{2}} \approx \frac{1}{\sqrt{2 \delta}}
$$

and the ratio between appropriate velocities is equal to the same amount

$$
\frac{U_{n \delta}}{U_{\delta}}=\frac{\lambda_{n \delta} f}{\lambda_{\delta} f} \approx \frac{1}{\sqrt{2 \delta}}
$$

\section{Two Flavor Neutrino Oscillations}

All analyses in the neutrino oscillation theory start from the phase factor which represents the only found for derivation the neutrino oscillation properties. are given as in [1,2,5-24].Thus, we shall look firstly, whether by the analyzing the phase factor of the plane wave we can come to the information about the speed of neutrino oscillations. In that sense we shall write wave function of the plane wave

$$
\begin{aligned}
& \Psi=A \exp \left[\frac{i}{\hbar}(p x-E t)\right] \\
& =A \exp (i \Phi)
\end{aligned}
$$

and then to single out the phase factor itself

$$
\Phi=\frac{1}{\hbar}(p x-E t)
$$

The velocity of propagation of de Broglie's wave one finds as the moving velocity of constant phase and it amounts to

$$
u=\frac{d x}{d t}=\frac{E}{p}=\frac{c^{2}}{v}
$$

Even though generally speaking velocity $v$ according to the theory of relativity can not be greater than the light speed, nevertheless as we see the phase speed (46) exceeds the light speed. Also, such monochromatic wave described, by equation (44) in principle could not transmit neither the particle alone, nor its energy according to the theory of relativity.

However, despite of such conclusion, unique theoretical approach for the explanation the oscillation phenomenon enfolds just via phase factor (45).And by such theoretical approach unambiguous one comes to the conclusion that the neutrino oscillations enfold with the speed greater than light speed (46).Now, there are not any limitations assigned by theory of relativity. Because, it is clear in accordance with (46) that such monochromatic wave transmit either the particle and energy. And about that can speak authoritative because the phenomenon of neutrino oscillation in the nature is experimentally confirmed. as in [27-39].

And of course, based on such consideration, it is quite clear that on the oscillations neutrinos is applicable tachyonic nature of neutrino.

Note that relation (46) coincides with relation which is derived as in [25].From that we conclude that neutrino speed measured in the laboratories assigned as $v$ that is really tachyon speed in view that is given by four-dimensional space-time which belongs to theory of relativity.

However, the real neutrino speed, which belongs to the tachyon four-dimensional space-time, one gets from just that relation which is mutual for either four-dimensional space-times, how it is explained as in [25]

$$
U=\frac{c^{2}}{v}=\frac{c^{2}}{[c /(1+\delta)]}=c(1+\delta)
$$

Comment: The fact itself that neutrino oscillations enfold via the phase, until the phase itself transmits with the speed greater the light speed, means that the transmission either particle and its energy enfold via monochromatic wave. As it is known from text-books of quantum mechanics that the wave packet was introduced as the represent of a particle as the wave, because the transmission either particle and its energy with the speed greater than the light speed is forbidden in the theory of relativity.

In the theory of relativity rest mass and rest energy correspond to the point at zero speed. Until, in the tachyon theory the point at the infinite velocity corresponds to the 
definitive momentum equal to the product of neutrino mass and speed of light as in [25].

\section{Klein-Gordon Equation for Neutrinos}

We shall carry out the proceeding as it was made for the special theory of relativity. So, we start with the identity from the tachyon theory describing the energy

$$
E=\frac{U^{2}}{c} \sqrt{p^{2}-m^{2} c^{2}}=\sqrt{p^{2} \frac{U^{4}}{c^{2}}-m^{2} U^{4}}
$$

Then, just inserting the quantum mechanical operator for momentum and energy yield the equation

$$
i \hbar \frac{\partial}{\partial t}=\sqrt{\left(-i \hbar \frac{\partial}{\partial x}\right)^{2} \frac{U^{4}}{c^{2}}-m^{2} U^{4}}
$$

However, this equation has not sense, because differential equation cannot be evaluated while under the square root sign. In addition this equation as it stands instead begin with the square of the above identity (48), i.e.,

$$
E^{2}=p^{2} \frac{U^{4}}{c^{2}}-m^{2} U^{4}
$$

which when quantized, gives

$$
\left(i \hbar \frac{\partial}{\partial t}\right)^{2} \Psi=\frac{U^{4}}{c^{2}}\left(-i \hbar \frac{\partial}{\partial x}\right)^{2} \Psi-m^{2} U^{4} \Psi
$$

and simplifying this equation which becomes

$$
\frac{\partial^{2}}{\partial t^{2}} \Psi-\frac{U^{4}}{c^{2}} \frac{\partial^{2}}{\partial x^{2}} \Psi-\frac{m^{2} U^{4}}{\hbar^{2}} \Psi=0
$$

\section{Free Particle Solutions}

The Klein-Gordon equation for a free particle can be written as equation (52).We look for plane wave solutions of the form

$$
\Psi(x, t)=\exp [i( \pm k x-( \pm \omega) t)]=\exp \left[\frac{i}{\hbar}( \pm p x \mp E t)\right]
$$

for some constant angular frequency $\omega \in R$ and wave number $k \in R^{3}$. Substitution gives the dispersion relation

$$
-\omega^{2}+\frac{U^{4}}{c^{2}}|k|^{2}=\frac{m^{2} U^{4}}{\hbar^{2}}
$$

Only separation of the parts with the positive and negative frequency in the relationship

$$
\omega= \pm \sqrt{\frac{U^{4}}{c^{2}}|k|^{2}-\frac{m^{2} U^{4}}{\hbar^{2}}}
$$

gives the equation which satisfies wave function (53).

Energy and momentum are seen to be proportional to $\omega$ and $\vec{k}$

$$
\begin{gathered}
\langle\hat{p}\rangle=\langle\Psi|-i \hbar \nabla| \Psi\rangle=\hbar \vec{k} \\
\langle E\rangle=\left\langle\Psi\left|i \hbar \frac{\partial}{\partial t}\right| \Psi\right\rangle=\hbar \omega
\end{gathered}
$$

So, dispersion relation is just the energetic function of tachyonic particle (5):

$$
\begin{aligned}
& \langle E\rangle^{2}=\frac{U^{4}}{c^{2}}\langle p\rangle^{2}-m^{2} U^{4} \\
& =\frac{U^{4}}{c^{2}}\left(\langle p\rangle^{2}-m^{2} c^{2}\right)
\end{aligned}
$$

For the case of the independence of time i.e., when is

$$
\frac{\partial}{\partial t} \Psi=0
$$

Klein-Gordon equation reduces to the equation

$$
\left(\frac{U^{4}}{c^{2}} \frac{\partial^{2}}{\partial x^{2}}+\frac{m^{2} U^{4}}{\hbar^{2}}\right) \Psi=0
$$

i.e.,

$$
\left(\frac{\partial^{2}}{\partial x^{2}}+\frac{m^{2} c^{2}}{\hbar^{2}}\right) \Psi=0
$$

Also, and in that case we look for plane wave solutions of the form

$$
\Psi(x)=\exp \left(i \frac{p x}{\hbar}\right)=\exp (i k x)
$$

where is square of wave number is:

$$
k^{2}=\frac{m^{2} c^{2}}{\hbar^{2}}
$$

And the particle momentum is:

$$
p= \pm \hbar k= \pm m c
$$

This momentum represents neutrino when its velocity is

$$
\begin{aligned}
& U \approx c(1+n \delta) \approx c \frac{1}{\sqrt{2 \delta}} \approx \frac{E}{m c} ; \\
& n \delta>>1
\end{aligned}
$$

Taking into account (62) and (63) wave function can be written in the generally form:

$$
\begin{aligned}
& \Psi(x)=\exp \left( \pm i \frac{m c}{\hbar} x\right) \\
& =\exp ( \pm i k x)
\end{aligned}
$$


where the sign "plus" is in relation with neutrinos, and sign „minus“ with antineutrinos, and it is apparently that the particle moving is along the $x$-axis.

\section{Why Neutrinos as Tachyons Mutually Oscillate}

In physics is well known fact that quantum of lightphotons do not oscillate as neutrinos do. Impossibility of the oscillations is connected with the zero phase of photon. It can be represented by the plane wave

$$
\Psi=\exp \left[\frac{i}{\hbar}\left(p_{p h} x-E t\right)\right]=\exp \left(i \Phi_{p h}\right)
$$

Let us look what is phase factor $\Phi_{p h}$ of photon. We know that the energy and the momentum of the photons are related by

$$
E=p_{p h} c
$$

and its traveling distance is

$$
x=c t
$$

Substitutions (68) and (69) within (67) give

$$
\Phi=p_{p h} c t-p_{p h} c t=0
$$

zero phase factor. It means that the photon oscillations are not allowed. The contribution to that conclusion is founded on the fact that the photons with equal energy can possess only one momentum, what is apparently from relation (68)

$$
E_{1}=p_{p h 1} c, E_{2}=p_{p h 2} c
$$

Namely, if

$$
\begin{aligned}
& E_{1}=E=p_{p h 1} c, \\
& E_{2}=E=p_{p h 2} c
\end{aligned}
$$

then photons with equal energy possess equal momentums. The phase velocity calculated from the phase factor (67) is

$$
v_{p h}=\frac{d x}{d t}=\frac{E}{p_{p h}}=c
$$

The phase factor of the tachyon is determined in the plane wave equation (53):

$$
\Phi=\frac{1}{\hbar}[(p x-E t)]
$$

For single tachyonic neutrino we can write

$$
x=U t ; E=p U
$$

Substitution (74) within (73) gives zero phase factor

$$
\Phi_{T}=\frac{1}{\hbar}[(p U t-p U t)]=0
$$

Zero phase factor cold mean that neutrino as tachyon particle do not capable to oscillate. However, despite of photons neutrinos with equal energy can possess different momentums and different velocities. It is apparently if we write the appropriate energy relations

$$
\begin{aligned}
& E_{1}=E=p_{1} U_{1}=m_{1} \Gamma_{1} U_{1}^{2} \\
& \Gamma_{1}=\frac{1}{\sqrt{\left(U_{1}^{2} / c^{2}\right)-1}} \\
& E_{2}=E=p_{2} U_{2}=m_{2} \Gamma_{2} U_{2}^{2} \\
& \Gamma_{2}=\frac{1}{\sqrt{\left(U_{2}^{2} / c^{2}\right)-1}}
\end{aligned}
$$

Neutrinos with different masses and velocities (76,76a) could mutually interfere and thus to realize the conditions for their flavor oscillations $\left(v_{e} \rightarrow v_{\mu}\right)$ and $\left(v_{\mu} \rightarrow v_{e}\right)$. If we take in account the relation

$$
p_{1} U_{1}=p_{2} U_{2}
$$

then,within the condition of equal-energy assumption, is evidently that come on to the changing of physical quantities of neutrinos: $p_{1} \rightarrow p_{2}, U_{1} \rightarrow U_{2}, m_{1} \rightarrow m_{2}$ and vice-versa. So, we have two types of neutrinos which are expressed by the appropriate Klein-Gordon monochromatic waves with equal energy and with different momentums and velocities. Despite of the starting zero phase of each tachyon itself, the resultant phase as the difference between those phases is a definitive quantity.. We note that the phase velocity of neutrino in the form of monochromatic wave coincides with the speed of moving particle

$$
\frac{d x}{d t}=\frac{E}{p}=U=\frac{c^{2}}{v}
$$

what is in accordance with the found condition which is derived for the phase velocity that the process of oscillations are enfolded with the speed greater than the speed of light.

\section{Approximate Relationships}

In order to come on to the approximate relationships for the necessity of the usage formula (73) we shall analyze the left section of energetic function $E$ close to the light speed when $m^{2} c^{2}$ is small compared to its typical momentum $p$.In that case one can expand $E$ in $m^{2} / p^{2}$

$$
\begin{aligned}
& E=\frac{U^{2}}{c} \sqrt{p^{2}-m^{2} c^{2}} \\
& =\frac{U^{2}}{c} p \sqrt{1-\frac{m^{2} c^{2}}{p^{2}}} \approx \frac{U^{2}}{c} p-\frac{m^{2} c U^{2}}{2 p}
\end{aligned}
$$


And, similarly, one can expand $p$ in $m^{2} / E^{2}$

$$
\begin{aligned}
& p=\frac{E}{U}=\frac{U p}{c} \sqrt{1-\frac{m^{2} c^{2}}{p^{2}}} \\
& =\frac{E}{c} \sqrt{1-\frac{m^{2} c^{2} U^{2}}{E^{2}}} \approx \frac{E}{c}-\frac{m^{2} c U^{2}}{2 E}
\end{aligned}
$$

In other view, we use

$$
\begin{aligned}
& p=\sqrt{\frac{c^{2}}{U^{4}} E^{2}+m^{2} c^{2}} \\
& =\frac{c}{U^{2}} E \sqrt{1+\frac{m^{2} c^{2} U^{4}}{E^{2} c^{2}}} \approx \frac{c}{U^{2}} E+\frac{m^{2} c U^{2}}{2 E}
\end{aligned}
$$

which is equivalent with (80).Namely, equating (80) with (81)

$$
\frac{E}{c}-\frac{m^{2} c U^{2}}{2 E}=\frac{c}{U^{2}} E+\frac{m^{2} c U^{2}}{2 E}
$$

one gets properly value for energy function for $\mathrm{E}$ (3).

\section{The Proceeding for Two Flavor Oscillations in the Case of Equal- Energy Assumption}

We shall consider two types of neutrinos and we shall investigate neutrino oscillations $\left(v_{e} \rightarrow v_{\mu} \rightarrow v_{e}\right)$.

We assume that two mass eigenstates $\left|v_{1}\right\rangle$ and $\left|v_{2}\right\rangle$ neutrino composing the initially produced flavor state $\left|v_{e}\right\rangle$ have the same energy, and that the spatial propagation of these mass eigenstates can be described by the following phase factors:

$$
\left|v_{1}\right\rangle=\exp \left[\frac{i}{\hbar}\left(p_{1} x\right)\right]=\exp \left(i \Phi_{1}\right)
$$

and

$$
\left|v_{2}\right\rangle=\exp \left[\frac{i}{\hbar}\left(p_{2} x\right)\right]=\exp \left(i \Phi_{2}\right)
$$

Anyway, equal energy means, all mass eigenstate are supposed to have the same energy, that is, $E_{1}=E_{2}=E$. Then the time dependence of the phase factor

$$
\begin{aligned}
& \exp \frac{i}{\hbar}\left[\left(p_{1}-p_{2}\right) x-\left(E_{1}-E_{2}\right) t\right] \\
& =\exp \frac{i}{\hbar}\left(p_{1}-p_{2}\right)=\exp (i \Phi)
\end{aligned}
$$

vanishes and the resulting phase factor becomes:

$$
\Phi(x)=\frac{1}{\hbar}\left[\left(p_{1}-p_{2}\right) x\right]
$$

and as it is seen it is justifiable to bring the mass eigenstates in the forms (83) and (84).

Neutrino of type $v_{e}$ (electron neutrino flavor) with momentum

$$
p_{1}=m_{1} \Gamma_{1} U_{1}=\frac{h}{\lambda_{1}}
$$

is an energy eigenstate with eigenvalue

$$
E_{1}=\frac{U_{1}^{2}}{c} \sqrt{p_{1}^{2}-m_{1}^{2} c^{2}}
$$

and neutrino of type $v_{\mu}$ (muon neutrino flavor) with momentum

$$
p_{2}=m_{2} \Gamma_{2} U_{2}=\frac{h}{\lambda_{2}}
$$

is an energy eigenstate with eigenvalue

$$
E_{2}=\frac{U_{2}^{2}}{c} \sqrt{p_{2}^{2}-m_{2}^{2} c^{2}}
$$

There two sets of states are related to each other by unitary mixing matrix which will be described in the next section.

Neutrino oscillations are experimental fact as in [27-39]. It means that neutrinos as tachyons are not isolated to each other, but they are always in the mutual connection; they interfere Using the solution of Klein-Gordon equation obtained as the plane wave we shall our further researches connect with neutrino oscillations.

A neutrino with different flavor generates at a source. It will be produced as a linear combination of two states of definite mass.

We shall now rewrite the standard proceeding analysing the case of two flavor neutrino oscillation. As it is known there is only one unitary matrix liken to the $2 \times 2$ rotation matrix which rotates a vector in the flavor basis into a vector in the mass basis. Our aim is to find different mixed state at $(x, t)$ using that $2 \times 2$ PMNS (Pontecorvo-Maka-NakagavaSakata) mixing matrix as in $[12,13,16,17,18,34]$

$$
M=\left(\begin{array}{cc}
\cos \theta & \sin \theta \\
-\sin \theta & \cos \theta
\end{array}\right)
$$

where $\theta$ is mixing angle. So, we can write the mass states at some space-time point $(x, t)$ as

$$
\begin{aligned}
& \left(\begin{array}{l}
\left|v_{1}(x, t)\right\rangle \\
\left|v_{1}(x, t)\right\rangle
\end{array}\right)=\left(\begin{array}{cc}
\exp \left(i \Phi_{1}\right) & 0 \\
0 & \exp \left(i \Phi_{2}\right)
\end{array}\right)\left(\begin{array}{l}
\left|v_{e}(x, t)\right\rangle \\
\left|v_{\mu}(x, t)\right\rangle
\end{array}\right) \\
& =\left(\begin{array}{cc}
\exp \left(i \Phi_{1}\right) & 0 \\
0 & \exp \left(i \Phi_{2}\right)
\end{array}\right) M^{-1}\left(\begin{array}{l}
\left|v_{e}(0,0)\right\rangle \\
\left|v_{\mu}(0,0)\right\rangle
\end{array}\right) \\
& =\left(\begin{array}{cc}
\exp \left(i \Phi_{1}\right) & 0 \\
0 & \exp \left(i \Phi_{2}\right)
\end{array}\right)\left(\begin{array}{cc}
\cos \theta & -\sin \theta \\
\sin \theta & \cos \theta
\end{array}\right) \\
& \left(\begin{array}{l}
\left|v_{e}(0,0)\right\rangle \\
\left|v_{\mu}(0,0)\right\rangle
\end{array}\right)
\end{aligned}
$$


Since we try to find the appropriate flavor $v_{\mu}(x, t)$ and $v_{e}(x, t)$ state at point $(x, t)$, then detection of neutrino is in relation with different mixed state at that point, and it is described by the following expression:

$$
\begin{aligned}
& \left(\begin{array}{l}
\left|v_{e}(x, t)\right\rangle \\
\left|v_{\mu}(x, t)\right\rangle
\end{array}\right)=M\left(\begin{array}{l}
\left|v_{1}(x, t)\right\rangle \\
\left|v_{1}(x, t)\right\rangle
\end{array}\right) \\
& =\left(\begin{array}{cc}
\cos \theta & \sin \theta \\
-\sin \theta & \cos \theta
\end{array}\right)\left(\begin{array}{ll}
\left|v_{1}(x, t)\right\rangle \\
\left|v_{1}(x, t)\right\rangle
\end{array}\right) \\
& =\left(\begin{array}{cc}
\cos \theta & \sin \theta \\
-\sin \theta & \cos \theta
\end{array}\right)\left(\begin{array}{cc}
\exp \left(i \Phi_{1}\right) & 0 \\
0 & \exp \left(i \Phi_{2}\right)
\end{array}\right) \\
& \left(\begin{array}{cc}
\cos \theta & -\sin \theta \\
\sin \theta & \cos \theta
\end{array}\right)\left(\begin{array}{l}
\left|v_{e}(0,0)\right\rangle \\
\left.v_{\mu}(0,0)\right\rangle
\end{array}\right)
\end{aligned}
$$

The oscillation probability of finding $\left|v_{\mu}\right\rangle$ in the case if the initially state is represented by pure $\left|v_{e}\right\rangle$ beam, one finds from relation

$$
\begin{aligned}
& P\left[v_{e} \rightarrow v_{\mu}\right]=\left|\left\langle v_{\mu}(x, t) \mid v_{e}(0,0)\right\rangle\right|^{2} \\
& =\sin ^{2} 2 \theta \sin ^{2} \frac{\Phi_{1}-\Phi_{2}}{2}
\end{aligned}
$$

and again of finding $\left|v_{e}(x, t)\right\rangle$ at the oscillation distance $x$ the appropriate probability will be:

$$
\begin{aligned}
& P\left[v_{e} \rightarrow v_{e}\right]=1-\left|\left\langle v_{\mu}(x, t) \mid v_{e}(0,0)\right\rangle\right|^{2} \\
& =1-\sin ^{2} 2 \theta \sin ^{2} \frac{\Phi_{1}-\Phi_{2}}{2}
\end{aligned}
$$

In our investigation as the crucial factor is the phase difference which is equal

$$
\begin{aligned}
& \Phi_{1}-\Phi_{2}=\frac{1}{\hbar}\left[\left(p_{1}-p_{2}\right) x-\left(E_{1}-E_{2}\right) t\right] \\
& =\frac{x}{\hbar}\left(p_{1}-p_{2}\right)
\end{aligned}
$$

in the case of equal- energy $E_{1}=E_{2}=E$ assumption.

The oscillation length $L_{\text {osc }}$ one finds from relation

$$
P\left[v_{e}(0,0) \rightarrow v_{e}\left(L_{o s c}, T\right)\right]=1
$$

From here one finds

$$
\frac{\Phi_{1}-\Phi_{2}}{2}=\pi
$$

i.e.

$$
\frac{\Phi_{1}-\Phi_{2}}{2}=\frac{L_{o s c}}{2 \hbar}\left(p_{1}-p_{2}\right)=\pi
$$

And definite oscillation formula is

$$
L_{\text {osc }}\left(p_{1}-p_{2}\right)=h
$$

Note: The case of equal -energy assumption gives as a result a simple product of the difference between the neutrino momentums and oscillation length and it is equal to Planck's constant. As it is seen from the relation (75), mathematical analysis for the zero starting phase factor could be useless, if they were taken as the starting value. But, because neutrino mutually interfere then we after mathematical transformation get the difference between their phase factor which represent the measurable quantity in the experiments (equation (98)).

\section{The Postulate of Neutrino Flavor Confinement}

In text-book of quantum mechanics states that the position and momentum of a particle cannot be simultaneously measured with arbitrarily high precision. There is a minimum for the product of the uncertainties of these two measurements

$$
\Delta p \Delta x>\frac{\hbar}{2}
$$

This is not a statement about the inaccuracy of measuring instruments, nor a reflection on the quality of experimental method; it arises from the wave properties inherent in the quantum mechanical description of nature.

The uncertainty principle contains implications about the energy that would be required to contain a particle within a given volume. The energy required to contain particles comes from the fundamental forces, and in particular the electromagnetic force provides the attraction necessary to contain electrons within atom, and the strong nuclear force provides the attraction necessary to contain particles within the nucleus.

But Planck's constant appearing in the uncertainty principle, determines the size of the confinement that can be performed by these forces.

We note $\Psi^{*} \Psi$.is the probability of finding the particle where $\Psi$ is wave function.

In order to determine the energy of confinement was used a gross approximation

$$
\Delta p \Delta x=h
$$

This relation we may write again:

$$
\Delta p \Delta x=h
$$

But in this relation $\Delta p$ does not have any more that sense of the uncertainty of the momentum in the Heisenberg's uncertainty relation but it represents the difference between momentums of neutrinos which mutually oscillate, and we write that in the form:

$$
\Delta p=p_{1}-p_{2}
$$


Until $\Delta x$ does not have any more that sense of the uncertainty of the position in the Heisenberg's uncertainty relation but it represents the oscillation length $L_{o s c}$ which is the distance between source and detector.

And now we define a confinement postulate

$$
\left(p_{1}-p_{2}\right) L_{o s c}=h
$$

It connects microscopic quantities either $p_{1}$ and $p_{2}$ with macroscopic distance $L_{\text {osc }}$.

This confinement postulate contains within it the oscillation probability

$$
\begin{aligned}
& P\left[v_{e} \rightarrow v_{e}\right]=\left|\left\langle v_{\mu}(x, t) \mid v_{e}(0,0)\right\rangle\right|^{2} \\
& =1-\sin ^{2} 2 \theta \sin ^{2}\left[\frac{1}{\hbar}\left(\frac{p_{1}-p_{2}}{2} L_{o s c}\right)\right]
\end{aligned}
$$

which implies that whole oscillation process combines two parts: $\quad\left(v_{e} \rightarrow v_{e}\right)(\equiv)\left(v_{e} \rightarrow v_{\mu}\right)(+)\left(v_{\mu} \rightarrow v_{e}\right)$.It means that within the macroscopic space surrounded by distance $L_{o s c}$ confines neutrino flavor state $\left|v_{\mu}\right\rangle$ if the initial flavor emitted from the source was $\left|v_{e}\right\rangle$.So, the confinement postulate shows the appearing of neutrino flavor $\left|v_{\mu}\right\rangle$ within distance of $L_{\text {osc }}$.

As we see definition of the confinement postulate is very simple: The product between the difference of neutrino momentums $\Delta p=p_{1}-p_{2}$ and the oscillation length $L_{o s c}$ is equal to Planck's constant.

From the confinement postulate immediately one finds length of the oscillations $L_{\text {osc }}$ :

$$
L_{o s c}=\frac{h}{p_{1}-p_{2}}
$$

In this formula we shall implement the approximate relationships

$$
\begin{aligned}
& p_{1} \approx \frac{E_{1}}{c}-\frac{m_{1}^{2} c U_{1}^{2}}{2 E_{1}} \\
& p_{2} \approx \frac{E_{2}}{c}-\frac{m_{2}^{2} c U_{2}^{2}}{2 E_{2}}
\end{aligned}
$$

Under the equal -energy assumption i.e., $E_{1}=E_{2}=E$ the first terms in the equations (108) and (109) vanish and difference between momentums will be defined by relationship

$$
p_{1}-p_{2} \approx \frac{c}{2 E}\left[m_{2}^{2} U_{2}^{2}-m_{1}^{2} U_{1}^{2}\right]
$$

Substitution (110) within (107) gives oscillation length

$$
L_{\text {osc }}=\frac{2 E h}{c\left[m_{2}^{2} U_{2}^{2}-m_{1}^{2} U_{1}^{2}\right]}
$$

where $h$ is Planck's constant.

\subsection{Standard Proceeding of the Confinement Calculation}

In this section we rewrite known proceeding in text-book of quantum mechanics which is related with the confinement calculation.

The following very approximate calculation serves to give an order of magnitude for the energies required to contain particles within a nucleus.

Assume nuclear size $\Delta x=10^{-14} \mathrm{~m}$

The following relation shows that Planck's constant determines the relationship between $\Delta x$ and $\Delta p=p$ and therefore the energy confinement one finds from momentum

$$
\Delta p=p=\frac{h}{\Delta x} \approx 10^{-20} \mathrm{kgm} / \mathrm{s}
$$

One more the energy confinement is in dependence of the particle mass.

Energy to confine electron is:

$$
E_{e}=\frac{p^{2}}{2 m_{e}}=3.77 G e v
$$

and energy to confine neutrino is:

$$
E_{v}=\frac{p^{2}}{2 m_{v}}>3.77 \times 10^{6} \mathrm{Gev}
$$

Because $m_{v}<10^{6} m_{e}$.

\subsection{Comment}

The energy confinement is about of thousand above the observed energies of nuclear processes indicating that the electron cannot be confined in the nucleus. Also, more it is valid for neutrino whose the energy confinement is much more than electron one.

But, after all those particles in the process of disintegration of nucleus be emitted in the surrounding space. The question is: where are they from, if them is forbidden to be in the stable nucleus, because their confinement energies are much more than the energies in nuclear processes?

There is in physics an experimental fact; at the moment when the nucleus decay, in the simplest case, it emits in the surrounding space photons and some particles between them there are an electron (positron) and antineutrino (neutrino).According to the confinement principle we see that such particles as an electron and a neutrino cannot be confined in the nucleus.Also, according to the laws of quantum mechanics before the nucleus decay electron was not a belongings as a part of that nucleus, already it is appeared at the moment of the nucleus decay, as a consequence of a process of the interaction with the physical 
vacuum as in [26].

Also, before the nucleus decay neutrino was not a belongings as a part of that nucleus, already it is appeared at the moment of the nucleus decay, as a consequence of a process of the interaction with dark energy and dark matter as in [40-43].

\section{The Ratio Between Neutrino Masses}

Starting with relation (111) we have

$$
\begin{aligned}
& m_{2}^{2} U_{2}^{2}-m_{1}^{2} U_{1}^{2}=\frac{2 h E}{L_{o s c} c} \rightarrow \\
& m_{2}^{2}\left(1+\delta_{2}\right)^{2}-m_{1}^{2}\left(1+\delta_{1}\right)^{2} \\
& =\frac{2 h E}{L_{o s c} c^{3}}
\end{aligned}
$$

Where $U_{1}=c\left(1+\delta_{1}\right) ; U_{2}=c\left(1+\delta_{2}\right)$.

Taking into account that $\delta_{1}<<1 ; \delta_{1}^{2}<<\delta_{1} ; \delta_{2}<<1 ; \delta_{2}^{2}<<\delta_{2}$ one gets the approximate relation between masses:

$$
\begin{aligned}
& m_{2}^{2}\left(1+\delta_{2}\right)^{2}-m_{1}^{2}\left(1+\delta_{1}\right)^{2} \\
& =\frac{2 h E}{L_{o s c} c^{3}} \rightarrow m_{2}^{2}-m_{1}^{2} \approx \frac{2 h E}{L_{o s c} c^{3}}
\end{aligned}
$$

From the following relations

$$
\begin{aligned}
& m_{2}^{2}\left(1+\delta_{2}\right)^{2}-m_{1}^{2}\left(1+\delta_{1}\right)^{2} \\
& \approx m_{2}^{2}\left(1+2 \delta_{2}\right)-m_{1}^{2}\left(1+2 \delta_{1}\right)= \\
& m_{2}^{2}\left(1+2 \frac{m_{2}^{2} c^{4}}{2 E^{2}}\right)-m_{1}^{2}\left(1+2 \frac{m_{1}^{2} c^{4}}{2 E^{2}}\right) \\
& =m_{2}^{2}-m_{1}^{2}+\frac{c^{4}}{E^{2}}\left(m_{2}^{4}-m_{1}^{4}\right) \\
& =\left(m_{2}^{2}-m_{1}^{2}\right)\left[1+2 \frac{c^{4}}{2 E^{2}}\left(m_{2}^{2}+m_{1}^{2}\right)\right] \\
& =\left(m_{2}^{2}-m_{1}^{2}\right) \\
& {\left[1+2 \frac{c^{4} m_{2}^{2}}{2 E^{2}}+2 \frac{c^{4} m_{1}^{2}}{2 E^{2}}\right]} \\
& =\left(m_{2}^{2}-m_{1}^{2}\right)\left[1+2\left(\delta_{2}+\delta_{1}\right)\right]
\end{aligned}
$$

follows a mathematical identity:

$$
\begin{aligned}
& m_{2}^{2}\left(1+2 \delta_{2}\right)-m_{1}^{2}\left(1+2 \delta_{1}\right) \\
& =m_{2}^{2}\left[1+2\left(\delta_{2}+\delta_{1}\right)\right] \\
& -m_{1}^{2}\left[1+2\left(\delta_{2}+\delta_{1}\right)\right]
\end{aligned}
$$

The approximate relation between masses can be written as follows:

$$
m_{2}^{2} \approx m_{1}^{2}+\frac{2 h E}{L_{o s c} c^{3}}
$$

i.e.,

$$
m_{2}=\sqrt{m_{1}^{2}+\frac{2 h E}{L_{o s c} c^{3}}}
$$

And from this the oscillation length is:

$$
L_{\text {osc }} \approx \frac{2 h E}{\left(m_{2}^{2}-m_{1}^{2}\right) c^{3}}
$$

From here follows $m_{2}>m_{1}$.

We especially underline that:

$$
m_{2}^{2}\left[1+2\left(\delta_{2}+\delta_{1}\right)\right]-m_{1}^{2}\left[1+2\left(\delta_{2}+\delta_{1}\right)\right]=\frac{2 h E}{L_{o s c} c^{3}}
$$

And from here one obtains more exact result than in (117):

$$
m_{2}^{2}=m_{1}^{2}+\frac{2 h E}{L_{o s c} c^{3}} \frac{1}{1+2\left(\delta_{2}+\delta_{1}\right)}
$$

So, we have

$$
m_{2}=\sqrt{m_{1}^{2}+\frac{2 h E}{L_{o s c} c^{3}} \frac{1}{1+2\left(\delta_{2}+\delta_{1}\right)}}
$$

and

$$
L_{o s c} \approx \frac{2 h E}{\left(m_{2}^{2}-m_{1}^{2}\right)\left[1+2\left(\delta_{2}+\delta_{1}\right)\right] c^{3}}
$$

\section{The Ratio between Neutrino Velocities}

In this case, also, we write the appropriate relations for masses and energies:

$$
\begin{aligned}
& m_{2}^{2} U_{2}^{2}-m_{1}^{2} U_{1}^{2}=\frac{2 h E}{L_{o s c} c} \rightarrow \frac{E^{2}}{\Gamma_{2}^{2} U_{2}^{2}}-\frac{E^{2}}{\Gamma_{1}^{2} U_{1}^{2}} \\
& =\frac{2 h E}{L_{o s c} c} \rightarrow \frac{E^{2}}{U_{2}^{2}}\left(\frac{U_{2}^{2}}{c^{2}}-1\right)-\frac{E^{2}}{U_{1}^{2}}\left(\frac{U_{1}^{2}}{c^{2}}-1\right)=\frac{2 h E}{L_{o s c} c} \rightarrow \\
& \frac{1}{c^{2}}-\frac{1}{U_{2}^{2}}-\frac{1}{c^{2}}+\frac{1}{U_{1}^{2}}=\frac{2 h}{L_{o s c} c E} \rightarrow \\
& \frac{1}{U_{1}^{2}}-\frac{1}{U_{2}^{2}}=\frac{2 h}{L_{o s c} c E} \rightarrow \\
& \frac{\delta_{2}-\delta_{1}}{1+2\left(\delta_{2}+\delta_{1}\right)}=\frac{h c}{L_{o s c} E} \rightarrow \\
& \delta_{2}-\delta_{1}=\frac{h c}{L_{o s c} E}\left[1+2\left(\delta_{2}+\delta_{1}\right)\right] \approx \frac{h c}{L_{o s c} E}
\end{aligned}
$$

So, we have

$$
\delta_{2} \approx \delta_{1}+\frac{h c}{L_{o s c} E}
$$

i.e., 


$$
U_{2} \approx U_{1}+\frac{h c^{2}}{L_{o s c} E}
$$

From here follows $U_{2}>U_{1}$.

And oscillation length is:

$$
L_{\text {osc }} \approx \frac{h c^{2}}{\left(U_{2}-U_{1}\right) E}
$$

\section{The Ratio between the Neutrino Momentums}

In this case are given the relations between appropriates masses and momentums, as follows:

$$
\begin{aligned}
& m_{2}^{2} U_{2}^{2}-m_{1}^{2} U_{1}^{2}=\frac{2 h E}{L_{\text {osc }} c} \rightarrow \frac{p_{2}^{2}}{\Gamma_{2}^{2}}-\frac{p_{1}^{2}}{\Gamma_{1}^{2}} \\
& =\frac{2 h E}{L_{\text {osc }} c} \rightarrow p_{2}^{2}\left(\frac{U_{2}^{2}}{c^{2}}-1\right)-p_{1}^{2}\left(\frac{U_{1}^{2}}{c^{2}}-1\right) \\
& \rightarrow \\
& \frac{p_{2}^{2} U_{2}^{2}}{c^{2}}-p_{2}^{2}-\frac{p_{1}^{2} U_{1}^{2}}{c^{2}}+p_{1}^{2} \\
& =\frac{2 h E}{L_{o s c} c} \rightarrow p_{1}^{2}-p_{2}^{2}=\frac{2 h E}{L_{o s c} c}
\end{aligned}
$$

Because

$$
E=p_{1} U_{1}=p_{2} U_{2}
$$

So, we have:

$$
p_{1}=\sqrt{p_{2}^{2}+\frac{2 h E}{L_{o s c} c}}
$$

And oscillation length is:

$$
L_{o s c}=\frac{2 h E}{\left(p_{1}^{2}-p_{2}^{2}\right) c}
$$

From here follows $p_{1}>p_{2}$.Initial neutrino flavor transforms into other one $\left(v_{e} \rightarrow v_{\mu}\right)$ when comes to the following changes:

- Neutrino mass: $m_{1}$ transforms into $m_{2} ; m_{2}>m_{1}$

- Neutrino velocity: $U_{1}$ transforms into $U_{2} ; U_{2}>U_{1}$

- Neutrino momentum: $p_{1}$ transforms into $p_{2} ; p_{2}<p_{1}$

- And for oscillation $\left(v_{\mu} \rightarrow v_{e}\right)$ all transformations are vice-versa.

\section{The Plane Wave or the Wave Packet}

In the theory of neutrino oscillations, it is evident:

- That the phase velocity of plane wave is greater than the speed of light, which is a well known fact from the textbooks of quantum mechanics.
- That the all analyses in the theory of oscillations are made through the phase angles with horizontal transition, i.e., transition with equal energy. Then, of course, there is no loss of energy.

- That the theoretical analyses through the plane waves and the wave packets give identical results.

- That the speed of the wave packet matches the speed of the particles movement in the four-dimensional spacetime of the special theory of relativity.

- Since, all the analyses in the theory of neutrino oscillations are made through the phase of the plane wave, then it could be concluded that the oscillations extend by the phase velocity, meaning, faster than the speed of light.

- The formula for the phase velocity of the plane wave $c^{2} / v$ in Einstein's theory of relativity should be equated with the phase speed of propagation in the tachyon fourdimensional space-time which amounts to $U$.

- In that case we shall have $v U=c^{2}$.

- Speed of the wave packets, according to special theory of relativity, is equal to $v$.

- In actual fact, in laboratories the speed of neutrinos is measured equal to what we states as $v$.

In fact, it is the speed of neutrino as tachyons which are measured in laboratories in four-dimensional space-time according to the theory of relativity and this value must be corrected by multiplying by a factor

$(1+\delta)^{2} v=(1+\delta)^{2}[c /(1+\delta)]=c(1+\delta)=U$ In order to obtain the actual speed at which such a wave packet moves in its tachyon four-dimensional space-time as in [25].

The neutrino presentations has been done through the wave packet as in [18-23] as it is considered that the neutrinos do not move faster than light. However, now it can be seen that it is unnecessary, because it is enough to display neutrinos by plane wave as the solution of the Klein-Gordon equation. This is because this wave enfolds both the particle and its energy at the same speed $U$.

\section{The Transformations between two Different Four-Dimensional Space- Time}

We just have derived some relations and we them write again as in [25]:

$$
\begin{aligned}
& U=v(1+\delta)^{2} \\
& \gamma=\Gamma U \\
& v U=c^{2}
\end{aligned}
$$

One more physical quantity which transforms from one to the other four-dimensional space-time is neutrino mass. The connection between neutrino mass $m_{E}$ which belongs to the four-dimension space-time of theory of relativity, and $m_{T}$ which belongs to the tachyon four-dimension space-time, 
we find from relation of equal energies

$$
\begin{aligned}
& m_{E} \gamma c^{2}=m_{T} \Gamma U^{2} \\
& c \sqrt{p^{2}+m_{E}^{2} c^{2}}=c \sqrt{p^{2}+m_{T}^{2} U^{2}} \\
& =\frac{U^{2}}{c} \sqrt{p^{2}-m_{T}^{2} c^{2}}
\end{aligned}
$$

Substitutions $(129,130,131)$ within $(132)$ give the ratio between masses

$$
\frac{m_{E}}{m_{T}}=\frac{U}{c}=1+\delta
$$

On the basis of relation (133) we see that neutrinos in different four-dimensional space-time do not possess the same mass.

Suppose we have neutrino with mass $m_{T}$. His momentum is determined by (6). Substitution (133) within $(6,124)$

$$
p_{T}=m_{T} \Gamma U
$$

gives

$$
\begin{aligned}
& p_{T}=m_{T} \Gamma U=\left(\frac{c}{U} m_{E}\right)\left(\gamma \frac{c}{U}\right) U \\
& =m_{E} \gamma \frac{c^{2}}{U}=m_{E} \gamma=p_{E}
\end{aligned}
$$

and vice-versa

$$
\begin{aligned}
& p_{E}=m_{E} \mathcal{N}=\left(\frac{U}{c} m_{T}\right)\left(\Gamma \frac{U}{c}\right)\left(\frac{c^{2}}{U}\right) \\
& =m_{T} \Gamma U=p_{T}
\end{aligned}
$$

Making the ratio between (135) and (136) we have:

$$
\begin{aligned}
& \frac{p_{E}}{p_{T}}=\frac{m_{E} \gamma}{m_{T} \Gamma U}=\left(\frac{m_{E}}{m_{T}}\right)\left(\frac{\gamma}{\Gamma}\right)\left(\frac{v}{U}\right) \\
& =\left(\frac{U}{c}\right)\left(\frac{U}{c}\right)\left(\frac{c^{2}}{U^{2}}\right)=1
\end{aligned}
$$

From relation (137) follows that neutrino momentums stay unchanged no matter in which systems they have observed.

Only crucial conclusion from this analysis is that in experimental measured neutrino mass in four-dimensional space-time of the special theory of relativity neutrino mass must be divided by factor $1+\delta$.So, really measured neutrino mass in the experiments is $m_{E}=m_{\exp }$ and it is connected with neutrino mass as tachyon particles over relation:

$$
m_{T}=m_{E} \frac{c}{U}=\frac{m_{\mathrm{exp}}}{1+\delta}
$$

Suppose we have tachyonic matrix

$$
T=\left(\begin{array}{cc}
\Gamma \beta_{T} & -\Gamma \\
-\Gamma & \Gamma \beta_{T}
\end{array}\right)=\left(\begin{array}{cc}
\Gamma \frac{U}{c} & -\Gamma \\
-\Gamma & \Gamma \frac{U}{c}
\end{array}\right)
$$

and we regard this matrix in the four-dimension space-time of the theory of relativity. Using (120), (121) and (122) it becomes

$$
\begin{aligned}
& T=\left(\begin{array}{cc}
\Gamma \frac{U}{c} & -\Gamma \\
-\Gamma & \Gamma \frac{U}{c}
\end{array}\right)=\left(\begin{array}{cc}
\gamma & -\gamma \frac{c}{U} \\
-\gamma \frac{c}{U} & \gamma
\end{array}\right) \\
& =\left(\begin{array}{cc}
\gamma & -\gamma \frac{c}{\left(c^{2} / v\right)} \\
-\gamma \frac{c}{\left(c^{2} / v\right)} & \gamma
\end{array}\right)=\left(\begin{array}{cc}
\gamma & -\gamma \frac{v}{c} \\
-\gamma \frac{v}{c} & \gamma
\end{array}\right) \\
& =\left(\begin{array}{cc}
\gamma & -\gamma \beta_{E} \\
-\gamma \beta_{E} & \gamma
\end{array}\right)=L
\end{aligned}
$$

and initial matrix $T$ transforms into Lorentz's matrix.

And vice-versa; Let us consider Lorentz's matrix within tachyon four-dimensional apace-time

$$
\begin{aligned}
& L=\left(\begin{array}{cc}
\gamma & -\gamma \beta_{E} \\
-\gamma \beta_{E} & \gamma
\end{array}\right)=\left(\begin{array}{cc}
\gamma & -\gamma \frac{v}{c} \\
-\gamma \frac{v}{c} & \gamma
\end{array}\right) \\
& =\left(\begin{array}{cc}
\Gamma \frac{U}{c} & -\Gamma \frac{U}{c} \frac{v}{c} \\
-\Gamma \frac{U}{c} \frac{v}{c} & \Gamma \frac{U}{c}
\end{array}\right)=\left(\begin{array}{cc}
\Gamma \beta_{T} & -\Gamma \\
-\Gamma & \Gamma \beta_{T}
\end{array}\right)=T
\end{aligned}
$$

and we see that it transforms into Tachyon matrix. We see that there is mutual transformation over the barrier which is defined by the speed of light $L \rightarrow T$ and $T \rightarrow L$.Jumping over light barrier tachyon matrix transforms into Lorentz's matrix, and vice- versa.

Let's write relation (10) again:

$$
E=c \sqrt{p^{2}+m_{T}^{2} U^{2}}
$$

Introducing relation (138) within tachyon relation (142) one gets immediately Einstein's relation

$$
\begin{gathered}
E=c \sqrt{p^{2}+m_{E}^{2} \frac{c^{2}}{U^{2}} U^{2}}=c \sqrt{p^{2}+m_{E}^{2} c^{2}} \\
E=p c \sqrt{1+\frac{1}{\Gamma^{2}}}=p U
\end{gathered}
$$

And substitutions relations (129) and (130) within (144) give: 


$$
\begin{aligned}
& E=p c \sqrt{1+\frac{1}{\Gamma^{2}}}=p c \sqrt{1+\frac{1}{[\gamma(c / U)]^{2}}} \\
& =p c \sqrt{1+\frac{1}{[\gamma(v / c)]^{2}}} \\
& =p c \sqrt{1+\frac{c^{2}}{v^{2}}\left(1-\frac{v^{2}}{c^{2}}\right)}=p \frac{c^{2}}{v}
\end{aligned}
$$

well known Einstein's energy-momentum relation.

Or, if we take relation (131) only, and if it introduces within (144) immediately follows

$$
E=p c \sqrt{1+\frac{1}{\Gamma^{2}}}=p U=p \frac{c^{2}}{v}
$$

\section{Comment on the Oscillation Phase}

Let's write the formulas for the oscillation probability for the case of two flavor oscillations.

Firstly, let's write the oscillation probability formula which is derived using the standard proceeding based on the special theory of relativity:

$$
\begin{aligned}
& P\left[v_{e} \rightarrow v_{e}\right]=1-\sin ^{2} 2 \theta \sin ^{2} \frac{\Phi_{1 E}-\Phi_{2 E}}{2} \\
& =1-\sin ^{2} 2 \theta \sin ^{2}\left[\frac{L c^{3}}{4 \hbar E}\left(m_{2 E}^{2}-m_{1 E}^{2}\right)\right]
\end{aligned}
$$

And the oscillation probability formula which is derived in accordance with the tachyon theory:

$$
\begin{aligned}
& P\left[v_{e} \rightarrow v_{e}\right]=1-\sin ^{2} 2 \theta \sin ^{2} \frac{\Phi_{1}-\Phi_{2}}{2} \\
& =1-\sin ^{2} 2 \theta \sin ^{2}\left[\frac{L c}{4 \hbar E}\left(m_{2 T}^{2} U_{2}^{2}-m_{1 T}^{2} U_{1}^{2}\right)\right]
\end{aligned}
$$

Let us consider now only factors in the angular bracket of relations (147) and (148).

In special theory of relativity we have:

$$
\frac{L}{2 \hbar}\left(p_{1}-p_{2}\right)=\frac{L c^{3}}{4 \hbar E}\left(m_{2 E}^{2}-m_{1 E}^{2}\right)
$$

In theory of tachyonic nature of neutrino we have:

$$
\frac{L}{2 \hbar}\left(p_{1}-p_{2}\right)=\frac{L c}{4 \hbar E}\left(m_{2 T}^{2} U_{2}^{2}-m_{1 E}^{2} U_{1}^{2}\right)
$$

Let us look what we shall get if relation (133) in the form

$$
m_{E}=m_{T} \frac{U}{c}=m_{T}(1+\delta)
$$

substitutes within relation (149)? That substitution gives:

$$
\begin{aligned}
& \frac{L}{2 \hbar}\left(p_{1}-p_{2}\right) \\
& =\frac{L c^{3}}{4 \hbar E}\left[m_{2 T}^{2}\left(1+\delta_{2}\right)^{2}-m_{1 T}^{2}\left(1+\delta_{1}\right)^{2}\right] \\
& =\frac{L c}{4 \hbar E}\left(m_{2 T}^{2} U_{2}^{2}-m_{1 T}^{2} U_{1}^{2}\right)
\end{aligned}
$$

relation (150) which represents the calculated tachyon phase factor.

\section{Conclusion}

De Broglie's neutrino relations have been derived in this paper in the spirit of tachyon neutrino theory. The analysis of the physical characteristics of the neutrino as the tachyon particle has been done and a formula derived for the oscillation length. By analyzing phase angle of the plane wave, we came to the conclusion hat the oscillations of neutrinos could be performed by speeds greater than the speed of light. Setting off from the application of Heisenberg's uncertainty relation in the micro-world ,as a postulate of neutrino confinement was introduced in the macroscopic area defined by the neutrino oscillation length as it is shown by relation (105).It represents an example how the physical characteristics of neutrino as a quantum mechanical particle, which belongs to the micro-world are connected with its length of oscillation as a macroscopic quantity which belongs to the macro-world. Based on the carried research on oscillation of neutrino as a tachyonic particle, one can contend that:

- Neutrino oscillations are possible at equal- energy assumptions.

- Neutrino oscillation would not be possible, if the neutrino momentums are equal (formulas (107) and (128).

- Neutrino oscillations would not be possible at the equal neutrino speeds (formula (124).

If all neutrino parameters were different $E_{1} \neq E_{2}, p_{1} \neq p_{2}, U_{1} \neq U_{2}$, then the oscillation of neutrinos would have to be realized by transitions from the higher energy levels to the lower ones. In this case, the difference in the energy $\Delta E=E_{1}-E_{2}$ would have to be emitted into the surrounding space in relation to electromagnetic energy, i.e., in the form of photons of light or emission of electronpositron pairs. This means that neutrinos would lose energy at oscillations. The question remains open under what circumstances, if any, neutrino oscillations occur with different energies, momentums and velocities?

In the theory of neutrino oscillations, it is evident:

- That the phase velocity of plane wave is greater than the speed of light, which is a well known fact from the textbooks of quantum mechanics.

- That the all analyses in the theory of oscillations are made through the phase angles with horizontal transition, i.e., transition with equal -energy. Then, of course, here is no loss of energy. 
- That the theoretical analyses through the plane waves and the wave packets give identical results.

- That the speed of the wave packet matches the speed of the particles movement in the four-dimensional spacetime of the special theory of relativity.

- Since, all the analyses in the theory of neutrino oscillations are made through the phase of the plane wave, then it could be concluded that the oscillations extend by the phase velocity, meaning, faster than the speed of light.

- The formula for the phase velocity of the plane wave $c^{2} / v$ in Einstein's theory of relativity should be equated with the phase speed of propagation in the tachyon fourdimensional space-time which amounts to $U$.

- In that case we shall have $v U=c^{2}$.

- Speed of the wave packets, according to special theory of relativity, is equal to $v$.

- In actual fact, in laboratories the speed of neutrinos is measured equal to what we states as $v$.

In fact, it is the speed of neutrinos as tachyons which are measured in laboratories in four-dimensional space-time according to the theory of relativity and this value must be corrected by multiplying by a factor

$(1+\delta)^{2} v=(1+\delta)^{2}[c /(1+\delta)]=c(1+\delta)=U$ In order to obtain the actual speed at which such a wave packet moves in its tachyon four-dimensional space-time as in [25].

The neutrino presentations has been done through the wave packet as it is considered that the neutrinos do not move faster than light. However, now it can be seen that it is unnecessary, because it is enough to display neutrinos by plane wave as the solution of the Klein-Gordon equation. This is because this wave enfolds both the particle and particle energy at the same speed $U$.

Special attention was paid to the confinement postulate which connects the physical properties of neutrinos as microparticle with macroscopic dimension of the neutrino oscillation length.

Based on the mutual agreement of results between the classical procedure in the theory of neutrino oscillations and the proposed confinement postulate, it can be seen that the introduction of a postulate whose formulation is extremely simple is reasonable and reads as follows: the product of the difference between neutrino momentums and the oscillation length is equal to the Planck's constant. This postulate suggests the existence of an interconnection, which is not random but essential, between the micro-world and the macro-world.

In the end, a mutual relationship between the special theory of relativity and the theory of tachyon neutrino has been demonstrated, which is established based on the transformation of Lorentz matrix into Tachyon matrix and vice-versa. By theoretical consideration, the equality between energy and momentum of the particles in both fourdimensional space-times has been reached. However, the masses of the neutrinos in these two four-dimension spacetimes are not mutually equal. Taking into account this fact, it is shown that the formulas of phase factor-oscillation phase, which belongs to different four-dimension space-times directly transform from one into the other, and vice-versa.

\section{References}

[1] E. Kh. Akhmedov and A. Yu.Smirnov, „Paradoxes of neutrino oscillations", arxiv:hep-ph/0905.1903 (2009).

[2] Y. Foli and Q. Y. Liu, "A paradox on quantum field theory of neutrino mixing and oscillations", JHEP 10 (2006), 048, arxiv:hep-ph/0604069,

[3] Z. B. Todorovic, ,A possible view of experimental evidences of the tachyonic nature of neutrino", Sc.BUP Timisoara, Tom 57(71),2, M-F, Pages 99-110 (2012)

[4] Z.. B. Todorovic, "Theory of tachyonic nature of neutrino", Fund. Journal of Modern Physics, volume 6, Issuess 1-2, 2013 , pages 17-47.

[5] Y. Grossman and H. Lipkin, "Flavor oscillation from a spatially localized source: A simple general treatment", Phys.Rev.D55 (1997), 2760-2767, arxiv:hep-ph/9607201,

[6] M. Blasam and G. Vitiello, "Remarks on the neutrino oscillation formula," arxiv:hep-ph/9907382v2 (1999)

[7] S. M.Bilenky, C. Giunti, and W. Grimus, "Phenomenology of neutrino oscillations", arhiv:hep-ph/9812360 (1999).

[8] Y. Takenchi, Y. Tazaki, S. Y. Tsai, and T. Yamazaki, „How do neutrino propagate? Wave packet treatment of neutrino oscillation, energy/momentum/velocity prescriptions of neutrino oscillations", arxiv.:hep-ph/9809558

[9] S. De Leo, G. Ducati, and P. Rotelli, „Remarks upon the mass oscillation formula“, Modern Physics Letters, A 15, 20572068 (2000), arxiv.org:hep-ph/9906460,

[10] M. C. Gonsales-Garcia, Y. Nir, "Neutrino masses and mixing: evidence and implications", CERN-TH/2002-21, arxiv: hepph/0202058 (2003).

[11] G. L .Fogli, E. Lisi, A. Marrone, D. Monatanino, A. Palazzo, and A. M.Rotunno, "Global of neutrino masses, mixing and phases: entering the era of leptonig CP violation searches", arxiv: hep-ph/1205.5254 (2012).

[12] C. Gianti, „Flavor neutrinos status“, arxiv:hep-ph/0402217 (2004).

[13] C. Gianti, „Theory of neutrinos oscillations“, arxiv:hep$\mathrm{ph} / 0409230 \mathrm{v} 1$ (2004).

[14] M. Blasone P. P. Pacho, and H. W. C. Tseng, "Neutrino oscillation from relativistic flavor current", arxiv:hep$\mathrm{ph} / 0212402 \mathrm{v} 43$ (2004)

[15] L. B. Okum and I. S. Tsukerman, "Comment on equal velocity assumptions for neutrino oscillation", arxiv:hep-ph/0007262

[16] H. Lipkin, "Stodolsky's theorem and neutrino oscillations phase-for pedestrians: Simple answers to confusing questions", Phys. Lett.B 042 (2006).

[17] H. J. Lipkin, "Quantum theory of neutrino oscillations for pedestrians-simple answers to confusing questions", arxiv:hep-ph/0505141v4 (2006).. 
[18] P. Hernandez, „Neutrino physics“, arxiv: 1010.4131 (2010).

[19] L. Stodolsky, „The unnecesary wave packet“, Phys.Rev.D58 (1998), 0360035, hep-ph/980238

[20] C. Ginuti, "Neutrino wave packets in quantum field theory", arxiv.org:hep-ph/02015014,

[21] S. Yang and Bo. Q. Ma, "Lorentz violation in threee family neutrinos oscillations", In.J.Mod.Phys..1A (2009), arxiv: hep$\mathrm{ph} / 0910.0897 \mathrm{v} 1$ (2009).

[22] D. V. Forero, M. Tortola, and J. M. F. Valle, "Global status of neutrno oscillation parameters after 2012", arxiv: 1205.4018 (2012).

[23] C. N. Leung, "Neutrino test and special relativity", arxiv:hep$\mathrm{ph} / 0002973 \mathrm{v} 2(2000)$.

[24] P. H. Chaukowski, and S. Pokorori, "Quantum corrections to neutrino masses and mixing angles", arxiv:hep-ph/0110249 (2001).

[25] Z. B. Todorovic, "Interpretation of experimentally measured neutrino velocities based on tachyon theory of neutrino", Fund. Journal of Modern Physics, volume 7, Issues 1, 2014, pages 933.

[26] Z. B. Todorovic, „Theory of the origin of the electron“, Sc.BUP Timisoara, Tom 50(65),2, M-F, Pages 102-110 (2006).

[27] R. Davis Jr., D. S. Harmer, and K. C. Hoffman (1968). "Search for Neutrinos from the Sun". Physical Review Letters 20: 1205. Bibcode: 1968PhRvL...20.1205D. doi:10.1103/PhysRevLett.20.1205.

[28] V. Gribov and B. Pontecorvo (1969). "Neutrino astronomy and lepton charge". Physics Letters B 28: 493. Bibcode: 1969PhLB...28...493G. doi: 10.1016/0370-2693(69)90525-5.

[29] J. Schechter, J.W.F. Valle (1980). "Neutrino Masses in SU (2) $x$ U (1) Theories". Physical Review D 22: 2227. Bibcode:1980PhRvD...22.2227S. doi:10.1103/PhysRevD.22.2227.

[30] S. Eidelman et al. (2004). "Particle Data Group - The Review of Particle Physics". Physics Letters B 592 (1). arxiv: astroph/0406663. Bibcode: 2004PhLB...592...1P. doi:10.1016/j.physletb.2004.06.001. Chapter 15: Neutrino mass, mixing, and flavor change Revised September 2005.

[31] M. Honda; Y. Kao; N. Okamura; T. Takeuchi (2006). "A Simple Parameterization of Matter Effects on Neutrino Oscillations". Arxiv: hep-ph/0602115 hep-ph.
[32] Daya Bay Collaboration (2012). "Observation of electronantineutrino disappearance at Daya Bay". Physical Review Letters 108 (17): 171803. arxiv: 1203.1669. Bibcode: 2012PhRvL.108q1803A.

doi:10.1103/PhysRevLett.108.171803.

[33] K. Nakamura et al. (2010). "Review of Particle Physics". Journal of Physics G37: 1. Bibcode: 2010JPhG...37g5021N. doi:10.1088/0954-3899/37/7a/075021.

[34] J. W. F. Valle (2006). "Neutrino physics overview". Journal of Physics: Conference Series 53 (1): 473. arxiv: hepph/0608101. Bibcode: 2006JPhCS...53...473V. doi:10.1088/1742-6596/53/1/031.

[35] R.N. Mohapatra and J. W. F. Valle (1986). "Neutrino Mass and Baryon Number Nonconservation in Superstring Models". Physical Review D 34 (5): 1642. Bibcode: 1986PhRvD...34.1642M. doi:10.1103/PhysRevD.34.1642.

[36] A. Kostelecký, S. Stuart (1994). "Nonlinear neutrino oscillations in the expanding universe". Phys. Rev. D 49: 1740-1757. Bibcode: 1994PhRvD...49.1740K. doi:10.1103/PhysRevD.49.1740.

[37] Gonzalez-Garcia; Nir (2002). "Neutrino Masses and Mixing: Evidence and Implications". Reviews of Modern Physics 75 (2): 345-402. arxiv: hep-ph/0202058. Bibcode: 2003RvMP...75..345G. doi:10.1103/RevModPhys.75.345.

[38] Maltoni; Schwetz; Tortola; Valle (2004). "Status of global fits to neutrino oscillations". New Journal of Physics 6: 122. arxiv: hep-ph/0405172. Bibcode: 2004NJPh...6...122M. doi:10.1088/1367-2630/6/1/122.Forero;

[39] Tortola; Valle (2012). "Global status of neutrino oscillation parameters after Neutrino-2012". Physical Review D 86: 073012. arxiv: 1205.4018. Bibcode: 2012PhRvD...86g3012F. doi:10.1103/PhysRevD.86.073012.

[40] H. K. Jassal, „Tachyon field in cosmology“, PRAMANAJournal of physics, vol.62, N8.3, 2004, pp.757-760.

[41] H. Farajoilahi, A.Paranpak, and G.F.Fadakar, „Interacting agegaraphi dark energy model in tachyon cosmology coupled to matter", arxiv:physics. gen -ph/ 1206.5796v3,2012.

[42] F. C. W.Davies, „Tachyonic dark matter“, arxiv:astro$\mathrm{ph} / 0403048,2014$.

[43] Z. Kersestzes and L. A. Gergely,“ Combined cosmological tests of a bivalent tachyonic dark enegy scalar field model", arxiv:astro-ph..CO/.1408.3736v1,2014. 